\title{
Fabrication of Nanoparticles of Silymarin, Hesperetin and Glibenclamide by Evaporative Precipitation of Nanosuspension for Fast Dissolution
}

\section{Kakran $\mathbf{M}^{1,2}$, Sahoo GN1, Lin Li*}

${ }^{1}$ School of Mechanical and Aerospace Engineering, Nanyang Technological University, 50 Nanyang Avenue, Singapore 639798

${ }^{2}$ Institute of Materials Research and Engineering, Agency for Science Technology and Research (ASTAR), 3 Research Link, Singapore 117602

\begin{abstract}
Evaporative precipitation of nanosuspension (EPN) was used to prepare nanoparticles of poorly water soluble drugs, namely silymarin (SLM), hesperetin (HSP) and glibenclamide (GLB), with the aim of improving their rate of dissolution. The original drugs and EPN prepared drug nanoparticles were characterized by scanning electron microscopy (SEM), differential scanning calorimetry (DSC) and dissolution tester. The particle sizes were found to be influenced by the drug concentration and the solvent to antisolvent ratio. The smallest average particle sizes obtained were $350 \mathrm{~nm}$ for SLM, $450 \mathrm{~nm}$ for HSP and $120 \mathrm{~nm}$ for GLB. The DSC study suggested that the crystallinity of EPN prepared drug nanoparticles was lower than the original drug. The dissolution rate of EPN prepared drug nanoparticles markedly increased as compared to original drug. The dissolution rate was increased by up to $95 \%$ for SLM nanoparticles, up to $90 \%$ for HSP and up to almost $100 \%$ for the GLB nanoparticles fabricated. From this study, it can be concluded that the EPN is an effective method to fabricate drug nanoparticles with enhanced dissolution rate.
\end{abstract}

Keywords: Evaporative precipitation of nanosuspension; nanoparticles; dissolution; glibenclamide; hesperetin; silymarin

\section{Introduction}

Today many of the drug entities chosen for further development are extremely hydrophobic, displaying low or negligible water solubility [1]. Poorly water-soluble drugs tend to be eliminated from the gastrointestinal tract before they get an opportunity to fully dissolve and be absorbed into the blood circulation. This results in low bioavailability and poor dose proportionality, which greatly hinders their clinical translations [2] reasoned that as about $65 \%$ of the human body is made up of water, a drug must have certain water solubility and thus, possess an acceptable bioavailability level. Therefore, poor water solubility of many drugs is one of the major obstacles in the development of highly potent pharmaceuticals. In the present study, we have used three poorly water soluble drugs, namely: silymarin, hesperetin and glibenclamide.

Silymarin and hesperetin are polyphenols. Silymarin, extracted from the seeds of milk thistle (Silibum marianum Gaertn), is a mixture of flavonolignans including silybin, silychristin, isosilybin, silydianin, taxifolin, and various derivatives of these components [3]. Among the isomers, silybin is the major and most active component and responsible for its pharmacological activity. [4,5] highlighted the role of silymarin as a hepatoprotector to treat liver injuries and chronic hepatitis. Its hepatoprotective effect is due to its anti-oxidant and anti-inflammatory properties as demonstrated by [6,7]. However, the main problem with silymarin is its poor oral bioavailability $(23-47 \%)$ which is attributable to its poor solubility [8] and low permeability as well as degradation in the gastrointestinal tract [9]. The solubility of silymarin in distilled water was reported to be $58 \mu \mathrm{g} / \mathrm{ml}$ at $25{ }^{\circ} \mathrm{C}$ and can be considered as a practically insoluble drug [10]. Hence, silymarin is required in a large dose to achieve therapeutic plasma levels. Hesperetin is a kind of flavonoid that exists ubiquitously in plants, fruits and flowers [11-13] have shown that hesperetin is a powerful radical scavenger that promotes cellular antioxidant defence-related enzyme activity. Besides acting as an antioxidant [14], hesperetin is also a potential anti-inflammatory [15] and anticancer agent [16]. In spite of this wide spectrum of pharmacological properties, hesperetin has a low bioavailability due to its poor aqueous solubility and slow dissolution rate in the aqueous gastro-intestinal fluids, which greatly restricts its use in therapy according to [17]. The solubility of hesperetin as reported by [18] is around $15 \mu \mathrm{g} / \mathrm{ml}$ at $25{ }^{\circ} \mathrm{C}$. Formulations that improve the solubility of this drug may, therefore, provide improved therapeutic options for patients to achieve higher oral bioavailability. Glibenclamide, on the other hand, is a second-generation sulfonylurea and is a widely employed oral hypoglycemic drug for the treatment of non-insulin-dependent diabetic patients (Type 2 diabetes). It causes hypoglycemia by stimulating release of insulin from pancreatic $\beta$ cells and by increasing the sensitivity of peripheral tissue to insulin [19]. Clinical trials for glibenclamide have shown its therapeutic effects to control glucose level [20]. Besides being antidiabetic, glibenclamide has been shown to exhibit in-vivo antiplatelet potential as well and thus, suggested to protect against thrombosis development [21]. Glibenclamide is known to have a poor bioavailability, which is attributed to poor dissolution in the body and hence it is not fully absorbed by the body. Although therapeutic effects of glibenclamide being used to treat diabetic patients show significant decrease of blood glucose [20], the drug remains highly insoluble in water (about 2-4 $\mu \mathrm{g}$ / $\mathrm{ml}$ ). Therefore improvements in the solubility and dissolution of the drug will greatly increase the absorption by the human body.

Introducing an upgraded or advanced formulation significantly lowers the time, risk and money expended in drug development, in contrast to developing completely new drugs. According to [22], a classical formulation approach for the poorly soluble drugs is to

*Corresponding author: Lin L, School of Mechanical and Aerospace Engineering Nanyang Technological University, 50 Nanyang Avenue, Singapore 639798, Tel: +65 6790 6285; E-mail: mlli@ntu.edu.sg

Received November 19, 2014; Accepted December 10, 2014; Published January 15,2015

Citation: Kakran M, Sahoo GN, Li L (2015) Fabrication of Nanoparticles of Silymarin Hesperetin and Glibenclamide by Evaporative Precipitation of Nanosuspension for Fast Dissolution. Pharm Anal Acta 6: 326. doi:10.4172/2153-2435.1000326

Copyright: $\odot 2015$ Kakran M, et al. This is an open-access article distributed under the terms of the Creative Commons Attribution License, which permits unrestricted use, distribution, and reproduction in any medium, provided the original author and source are credited. 


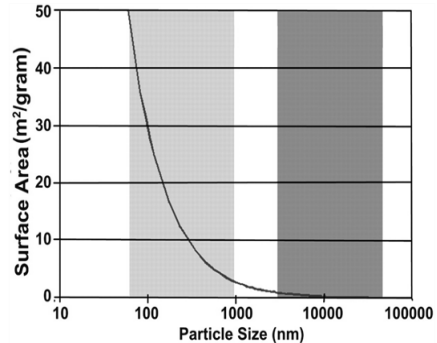

Figure 1: Reducing the particle size results in greater surface area per unit mass.

prepare smaller drug particles. [23] Have agreed and stated that the intention for reducing the particle size is to enlarge the surface area of the drug particles available for dissolution. At present, several new drug candidates possess very poor solubility and micronization does not bring those drugs to achieve satisfactory enhancement in the bioavailability. Subsequently, the choice is to go from micronization to nanonization to produce drug nanoparticles. The very small particle size results in a large surface area (Figure 1) and thus in an increased dissolution rate. As predicted by the [24] model of dissolution wherein the surface area of the drug is directly proportional to its rate of dissolution, drug particles in the nanometer size range will dissolve much more rapidly than a conventional formulation. Besides faster drug dissolution, nanoparticles also exhibit some interesting surface properties due to their very small size as emphasized by [25]. Particles having a size below $100 \mathrm{~nm}$ can evade mechanical filtration from the blood stream and accumulation in liver, spleen and kidneys, resulting in longer blood circulation times. Nanoparticles are able to deliver active pharmaceutical ingredients across a number of biological barriers, i.e., the blood-brain barrier [26], different types of mucosa [27] and epithelia [28], and cell membranes for transfection applications. Nanoparticles also show excellent adhesion to biological surfaces such as the epithelial gut wall, which is an advantage for sustained drug delivery. Positive effects of nanoparticles can be obtained for a wide range of drugs having diverse structures, molecular weights, steric groups and solubilities. Moreover, employing drug nanoparticles presents an added benefit of elevated mass per volume loading, which is important when a high dosage is needed. Such cases often fail with approaches involving molecular complexation (for example, cyclodextrins) because a high molar ratio of complexing excipient must be used. Fast dissolution of nanoparticles inspires us to fabricate a drug into nanoparticles so the drug will dissolve quickly.

In this study, the method called evaporative precipitation of nanosuspension (EPN) was used to fabricate drug nanoparticles. It is a new method which relies on high supersaturation and homogeneous nucleation to obtain drug nanoparticles. In this process, after adding hexane as an antisolvent to the drug solution, a uniform nanosuspension was obtained, which was independent of the agitation speed and flow rate. Filtering such a nanosuspension did not yield any particles as they were too small. Therefore, the EPN process was devised to extract the drug particles from the nanosuspension. The lower boiling point and heat of vaporization of hexane made it possible to quickly evaporate the drug nanosuspension to obtain the drug nanoparticles. The two process parameters studied were drug concentration in the solution and the solvent to antisolvent (SAS) ratio. Although the EPN method requires use of organic solvents, in our previous study we have shown that the amount of the residual solvents (ethanol and hexane) in the samples prepared by EPN was below the acceptable level for residual solvents in pharmaceuticals as determined by FDA for the safety of the patients [29]. For example, in the quercetin samples prepared the content of hexane was below $125 \mathrm{ppm}$ and that of ethanol was below 20 ppm as determined by gas chromatography. According to FDA, hexane is a Class 2 solvent, whose content should be limited $(\leq 290 \mathrm{ppm})$ and ethanol is a Class 3 solvent with a minimum content of 5,000 ppm. EPN represents an efficient method to obtain uniform drug nanoparticles with a significantly higher rate of dissolution in-vitro and hence, is used in the present study to fabricate drug nanoparticles. The present study focuses on fabrication of nanoparticles of poorly water soluble drugs: silymarin, hesperetin and glibenclamide by the EPN method.

\section{Materials and Methods}

\section{Materials}

Silymarin (SLM), hesperetin (HSP) and glibenclamide (GLB) were purchased from Sigma Aldrich Singapore. Organic solvents such as ethanol (99.9\%), acetone, methanol and hexane were obtained from Merck Singapore. Deionized (DI) water (Milli-Q, Millipore Singapore) was used.

\section{Fabrication Method}

Pure drug powder was dissolved in a solvent to prepare a $5-15 \mathrm{mg} / \mathrm{ml}$ drug solution. SLM was dissolved in acetone, HSP in ethanol and GLB in methanol respectively. Later the antisolvent (hexane) was added so as to get a nanosuspension. The ratio of solvent to antisolvent was varied from 1:10 to 1:20. For this process, the condition is that the solvent and the antisolvent should be miscible. The solvents used such as ethanol and acetone, were miscible with hexane. For methanol, although it is immiscible with hexane at 1:1 ratio but in our experiments using methanol to hexane ratio of 1:10 to 1:20 they formed homogeneous solutions, as has been reported earlier by [30].

Later, the drug nanosuspension prepared in a round bottom flask was attached to a rotary evaporator. The flask was immersed partly in water bath at $40^{\circ} \mathrm{C}$ and set to a rotation of $90 \mathrm{rpm}$. The evaporation occurred at $300 \mathrm{mbar}$. The solid in the flask after evaporation was vacuum dried overnight at $40^{\circ} \mathrm{C}$ and the solid particles in the flask were collected for further analysis. Parameters such as drug concentration in solution and solvent to antisolvent (SAS) ratio were optimized.

\section{Characterization}

Scanning Electron Microscopy (SEM): The morphology of samples was observed using a scanning electron microscope (JSM6390LA-SEM, Jeol Co., Tokyo, Japan). The powder samples were spread on a SEM stud and sputtered with gold before the SEM observations. The analysis of the particle size was performed using the UTHSCSA ImageTool program. Five or six high resolution and high magnification SEM pictures representative of the sample were used to find the particle size. The software was used to measure the average size of the particles seen in each of the SEM picture.

Differential Scanning Calorimetry (DSC): Differential scanning calorimetric (DSC) measurements were carried out using a TA DSC 200 thermal analyzer in a temperature range of $25-250^{\circ} \mathrm{C}$ at a heating rate of $10^{\circ} \mathrm{C} / \mathrm{min}$ in nitrogen gas. About 5 to $10 \mathrm{mg}$ of the sample mass was sealed in a closed aluminium pan for the DSC scan and an empty aluminum pan was used as the reference pan. The melting point and heat of fusion were calculated using the Universal Analysis (TA Q Series Advantage) software. 
Citation: Kakran M, Sahoo GN, Li L (2015) Fabrication of Nanoparticles of Silymarin, Hesperetin and Glibenclamide by Evaporative Precipitation of Nanosuspension for Fast Dissolution. Pharm Anal Acta 6: 326. doi:10.4172/2153-2435.1000326
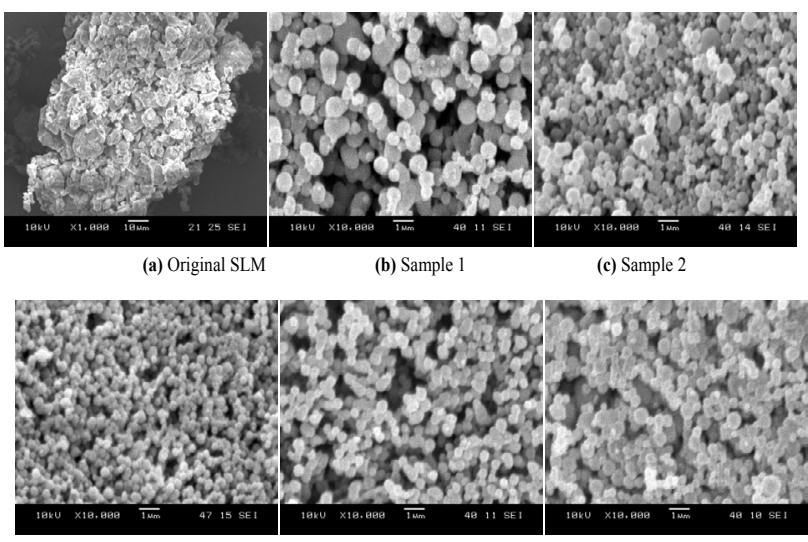

(d) Sample 3

(e) Sample 4

(f) Sample 5

Figure 2: SEM photographs of SLM particles prepared by EPN (refer to Table 1 for sample description).

\begin{tabular}{|c|c|c|c|c|c|c|c|}
\hline Samples & Drug Conc. & SAS & Particle Size (nm) & $\Delta \mathrm{H}_{\mathrm{f}}(\mathrm{J} / \mathrm{g})$ & Dissolution (\%) & $K\left(h^{-1}\right)$ & $R^{2}$ \\
\hline \multicolumn{3}{|c|}{ Original SLM } & 15,970 & 175.2 & $21.9 \pm 2.2$ & 0.058 & 0.84 \\
\hline 1 & 5 & $1: 10$ & 670.8 & 99.3 & $78.2 \pm 4.1$ & 0.345 & 0.85 \\
\hline 2 & 5 & $1: 15$ & 590.7 & 95.6 & $80.5 \pm 3.9$ & 0.358 & 0.84 \\
\hline 3 & 5 & $1: 20$ & 350.2 & 74.8 & $95.2 \pm 3.2$ & 0.646 & 0.900 \\
\hline 4 & 10 & $1: 20$ & 465.0 & 81.2 & $93.6 \pm 3.8$ & 0.604 & 0.93 \\
\hline 5 & 15 & $1: 20$ & 530.4 & 88.5 & $90.3 \pm 3.3$ & 0.518 & 0.94 \\
\hline \multicolumn{3}{|c|}{ Original HSP } & 34,000 & 302.0 & $4.72 \pm 1.0$ & 0.011 & 0.71 \\
\hline 1 & 5 & $1: 10$ & 890.3 & 240.6 & $60.0 \pm 3.0$ & 0.218 & 0.74 \\
\hline 2 & 5 & $1: 15$ & 720.6 & 190.1 & $75.6 \pm 3.1$ & 0.325 & 0.71 \\
\hline 3 & 5 & $1: 20$ & 450.2 & 147.9 & $90.0 \pm 3.2$ & 0.516 & 0.79 \\
\hline 4 & 10 & $1: 20$ & 545.4 & 140.1 & $85.9 \pm 3.0$ & 0.443 & 0.75 \\
\hline 5 & 15 & $1: 20$ & 600.7 & 137.7 & $84.0 \pm 2.9$ & 0.417 & 0.73 \\
\hline \multicolumn{3}{|c|}{ Original GLB } & 111,200 & 287.0 & $10.9 \pm 2.0$ & 0.027 & 0.85 \\
\hline 1 & 5 & $1: 10$ & 226.0 & 165.2 & $83.6 \pm 4.2$ & 0.462 & 0.96 \\
\hline 2 & 5 & $1: 15$ & 185.2 & 110.9 & $90.0 \pm 4.5$ & 0.605 & 0.95 \\
\hline 3 & 5 & $1: 20$ & 120.0 & 84.6 & $99.6 \pm 4.0$ & 1.345 & 0.991 .007 \\
\hline 4 & 10 & $1: 20$ & 140.3 & 93.7 & $98.1 \pm 2.2$ & 1.007 & 0.99 \\
\hline 5 & 15 & $1: 20$ & 150.1 & 99.1 & $96.3 \pm 3.2$ & 0.845 & 0.98 \\
\hline
\end{tabular}

Table 1: Preparation parameters namely drug concentration $(\mathrm{mg} / \mathrm{ml})$ and solvent to antisolvent ratio (SAS), and properties like mean particle size, melting enthalpy $(\triangle \mathrm{H})$ and the dissolution parameters: percent dissolution (at 4 hours), dissolution rate constant $K\left(h^{-1}\right)$ and the correlation coefficient $R^{2}$ of the original drugs (SLM, HSP and GLB) and their nanoparticles prepared by EPN.

Dissolution rate: The in vitro dissolution of the samples was determined using the paddle method (USP apparatus II) (Verkin Dissolution Tester DIS 8000) in $900 \mathrm{~mL}$ of DI water. The paddle rotation was set at $100 \mathrm{rpm}$ and the temperature was maintained at $37 \pm 0.5^{\circ} \mathrm{C}$. The dry powder samples weighing about $50 \mathrm{mg}, 10 \mathrm{mg}$ and $5 \mathrm{mg}$ for SLM, HSP and GLB respectively, were added to the dissolution vessel. About $1 \mathrm{~mL}$ of the dissolution media was collected at regular intervals and filtered for further analysis. For each sample the dissolution test was repeated for 3 times and the dissolution data were then averaged.

UV spectroscopy: The concentrations of drugs were analyzed using a UV spectrophotometer (UV-2102, Shanghai Instrument Ltd, China). SLM, HSP and GLB were analyzed at the detection wavelength of 286 $\mathrm{nm}, 323 \mathrm{~nm}$ and $299 \mathrm{~nm}$, respectively.

\section{Mathematical modeling of release kinetics}

The Noyes - Whitney equation provides a general guideline as to how the dissolution rate of an insoluble drug might be improved by reducing the particle size of a solid drug. The dissolution rate equation based on mass is expressed as follows:

$$
\frac{d m}{d t}=K\left(M_{S}-m\right)
$$

Where $m$ is the dissolution amount of a drug at time $t, d m / d t$ is the dissolution rate, $M_{s}$ is the dissolution amount at infinite time, and $t$ is the dissolution time. Integrating Eq. 1 with the initial condition of $m=$ 0 for $t=0$, then Eq. 2 is obtained.

$$
m=M_{S}[1-e(-K t)]
$$

Diving both sides by $M_{s}$, and we get Equation 3:

$$
\frac{m}{M_{S}}=[1-e(-K t)]
$$

Here, the dissolution rate constant $\mathrm{K}$ is defined as $\mathrm{AD} / \mathrm{h}$, where $\mathrm{A}$ is the surface area available for dissolution, $\mathrm{D}$ is the diffusion coefficient of the drug, and $\mathrm{h}$ is the thickness of the diffusion boundary layer adjacent to the surface of the dissolving drug. 


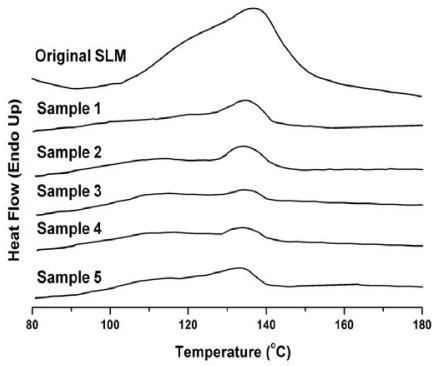

Figure 3: DSC thermogram of the original SLM and samples prepared by EPN.

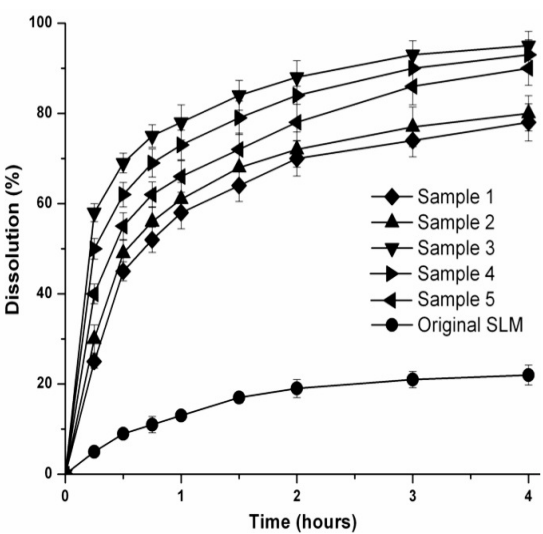

Figure 4: Dissolution profiles of original SLM and samples prepared by EPN.

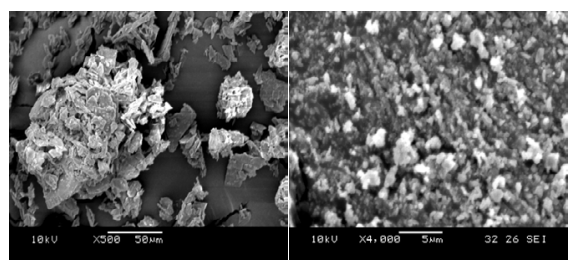

a) Original HSP

(b) Sample 1

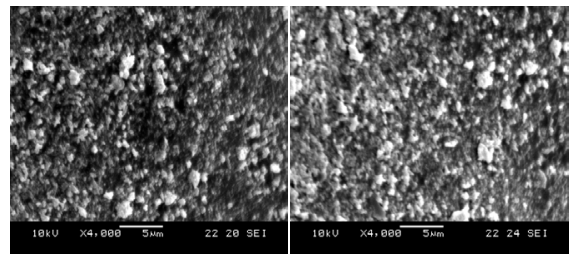

(c) Sample 3

(d) Sample 5

Figure 5: SEM photographs of HSP particles prepared by EPN (refer to Table 1 for sample description)

\section{Result and Discussion}

\section{Silymarin (SLM)}

As seen from Figure 2 and Table 1, original SLM powder exhibited the irregular shape and much bigger size $(15.97 \mu \mathrm{m})$ than the SLM nanoparticles prepared by the EPN method. It can be observed from Figure 2 that the SLM particles prepared by EPN were uniform and small $(350 \mathrm{~nm}$ to $670 \mathrm{~nm})$. The process parameters for all the samples prepared are listed in Table 1 along with the resulting particle sizes. The sample 3 exhibited the smallest particle size of $350.2 \mathrm{~nm}$. Decreasing the drug concentration and increasing the SAS ratio resulted in smaller particle size. This observation can be interpreted by the formation of nanoparticles through homogeneous nucleation. A higher initial concentration and larger solvent to antisolvent ratio may lead to greater supersaturation, which favors the formation of a large number of nuclei [31]. A larger number of nuclei mean smaller sized nuclei. Although a high initial concentration helps in greater supersaturation, it also has the opposite effect of causing aggregation of particles. In our case, the aggregation effect dominates and hence, a lower drug concentration produces smaller particles. Once nuclei are formed, growth occurs simultaneously. For the subsequent growth a large solvent to antisolvent ratio increases the diffusion distance for growing species and consequently molecular diffusion becomes the limiting step for nuclei growth as also observed in our previous studies [32,33].

In order to understand the effect of the fabrication method on the thermal properties of SLM, DSC measurements were conducted. The DSC thermograms (Figure 3) and the melting enthalpy $\Delta \mathrm{H}_{\mathrm{f}}$ values are given in Table 1. The original SLM had a melting peak at $137{ }^{\circ} \mathrm{C}$ and a melting enthalpy of $175 \mathrm{~J} / \mathrm{g}$, whereas the EPN prepared SLM nanoparticles had the melting peaks around $\sim 134-135{ }^{\circ} \mathrm{C}$ and the melting enthalpy was lower than that of the original SLM. From these results it can be concluded that the crystallinity of the SLM particles prepared by the EPN method was reduced compared to the original SLM powder.

The dissolution tests were also performed for the original SLM and the EPN prepared samples and their dissolution profiles (Figure 4). The dissolution of the EPN prepared samples was much faster than the original SLM. Only about $21.9 \%$ of the original SLM dissolved within 4 hours as compared to $78 \%$ - 95\% of dissolution for the EPN prepared samples. The sample 3 with the smallest particle size showed the greatest increase ( $\sim 5$ folds) in the dissolution rate of SLM compared to the original SLM powder. This improvement in the dissolution rate is attributed to the reduction in the particle size of SLM particles prepared by the EPN method.

The dissolution rate constant $K$ for the original drug and the nanoparticles prepared by EPN was obtained according to Equation 3. The calculated dissolution rate constant $K$, along with the correlation coefficient $R^{2}$, are listed in Table 1 . The $R^{2}$ value of more than 0.8 indicated good correlation between the Noyes-Whitney equation and our measured dissolution data. The value of dissolution rate constant $K$ increased with the decrease in the particle size. According to the NoyesWhitney equation, the dissolution rate of a drug can be increased by reducing the particle size to increase the particle's surface area available for dissolution. An increase in dissolution velocity and saturation solubility can also be achieved by changing the crystalline state of the material (e.g. from crystalline to amorphous or partially amorphous) [34]. Proposed that an amorphous or metastable form will dissolve at a faster rate because of its higher internal energy and greater molecular motion, as compared to crystalline materials. Our DSC study revealed that the crystallinity of SLM was reduced after the preparation by the EPN method, which also contributed to a faster rate of dissolution for the SLM nanoparticles.

\section{Hesperetin (HSP)}

The preparation conditions and properties of the original HSP powder and the HSP samples prepared by EPN are listed in Table 1. It can be observed from Figure 5 that as the SAS ratio increased from 1:10 (sample 1) to 1:20 (sample 3), the particl e size decreased from 
Citation: Kakran M, Sahoo GN, Li L (2015) Fabrication of Nanoparticles of Silymarin, Hesperetin and Glibenclamide by Evaporative Precipitation of Nanosuspension for Fast Dissolution. Pharm Anal Acta 6: 326. doi:10.4172/2153-2435.1000326

Page 5 of 7

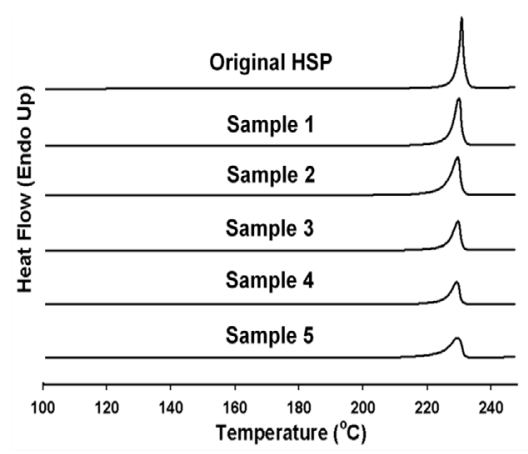

Figure 6: DSC thermogram of original HSP and samples prepared by EPN.

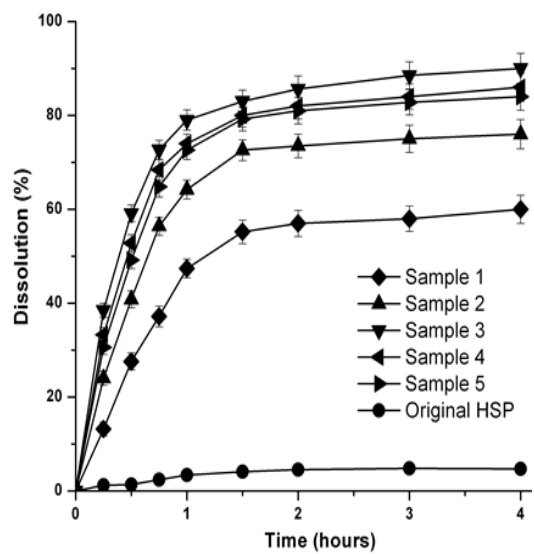

Figure 7: Dissolution profiles of original HSP, and samples prepared by EPN.

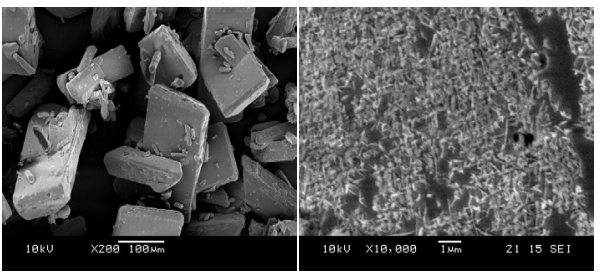

(a) Original GLB

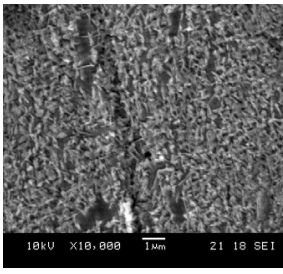

(c) Sample 3 (b) Sample 1

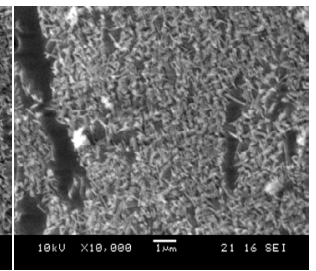

(d) Sample 5
Figure 8: SEM photographs of GLB particles prepared by EPN (refer to Table 1 for sample description).

$890 \mathrm{~nm}$ to $450 \mathrm{~nm}$, and it increased to $600 \mathrm{~nm}$ as drug concentration increased from $5 \mathrm{mg} / \mathrm{ml}$ (sample 3) to $15 \mathrm{mg} / \mathrm{ml}$ (sample 5). The DSC thermograms of the original HSP and its particles prepared by the EPN (Figure 6) and the melting enthalpy $\left(\Delta \mathrm{H}_{\mathrm{f}}\right)$ obtained from the DSC study are summarized in Table 1. The original HSP used in this study had a sharp melting endothermic peak at $230^{\circ} \mathrm{C}$ and a melting enthalpy of 302 $\mathrm{J} / \mathrm{g}$. It can also be observed that the EPN prepared HSP nanoparticles had a melting point of about $230^{\circ} \mathrm{C}$, very similar to the original HSP, but the melting enthalpy was lower than that of the original HSP. These results suggested that the crystallinity of HSP was decreased after it was prepared by the EPN method.

Next, the dissolution properties of the HSP samples were studied by the dissolution test. As seen from the dissolution profiles of the samples in Figure 7, only about $4.7 \%$ of the original HSP dissolved within 4 hours as compared to 60 to $90 \%$ of dissolution for the EPN prepared HSP samples. This represents a 12 to 20 times increase in the percent dissolution of HSP. In accordance with the Noyes-Whitney equation, the dissolution rate constant $K$ increased with the decrease in the particle size as seen from Table 1 . The best dissolution was shown by sample 3 , which was attributed to the maximum reduction in particle size which led to an increase in the exposed surface area available for dissolution. The DSC study revealed that the crystallinity of EPN prepared HSP nanoparticles was lower than that of the original HSP, which also contributed to the faster rate of dissolution for the HSP nanoparticles prepared.

\section{Glibenclamide (GLB)}

The effects of drug concentration and solvent to antisolvent (SAS) ratio on the fabrication of GLB nanoparticles by the EPN process were also examined as described in Table 1. The SEM microphotographs of the EPN prepared GLB particles under variable conditions (Figure 8). It is observed from Figure 8 that the EPN prepared GLB particles were in the size range of $120-226 \mathrm{~nm}$. The EPN prepared drug nanoparticles were more uniform and the uniformity was more prominent at a higher solvent to antisolvent ratio. The size of GLB nanoparticles tended to decrease at lower drug concentration and higher solvent to antisolvent ratio. In order to understand the effect of the EPN process on the thermal properties of GLB, DSC measurement was conducted and the melting heat $\left(\Delta \mathrm{H}_{\mathrm{f}}\right)$ values obtained from the DSC study are summarized in Table 1. The heat of fusion of the original GLB powder was higher than that of the EPN prepared GLB particles. These results suggest that the crystallinity of GLB particles was decreased when the particles were prepared by the EPN method. Table 1 shows the experimental data for the dissolution of the original GLB powder and EPN prepared GLB particles, and the dissolution profiles for the samples (Figures 9 and 10 ). The dissolution of original GLB powder in water was very low so that after 4 hours only about $10.9 \%$ of the original GLB powder was dissolved. For the EPN prepared samples the dissolution rate was better. The best dissolution was observed for the EPN prepared sample 3, with $99.6 \%$ drug dissolved after 4 hours. This is an approximately 9 times

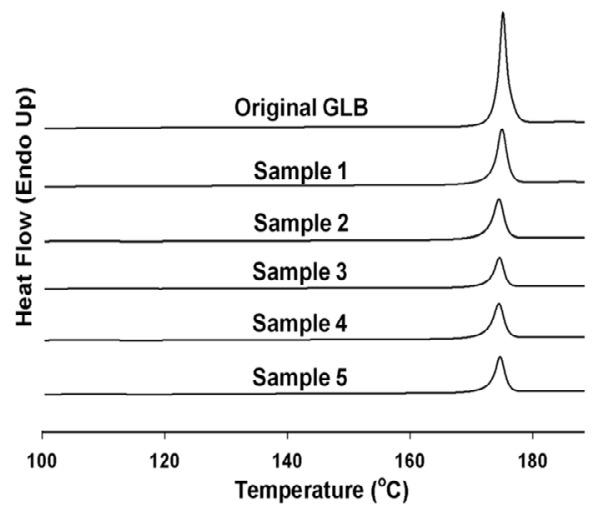

Figure 9: DSC thermogram of original GLB and samples prepared by EPN. 


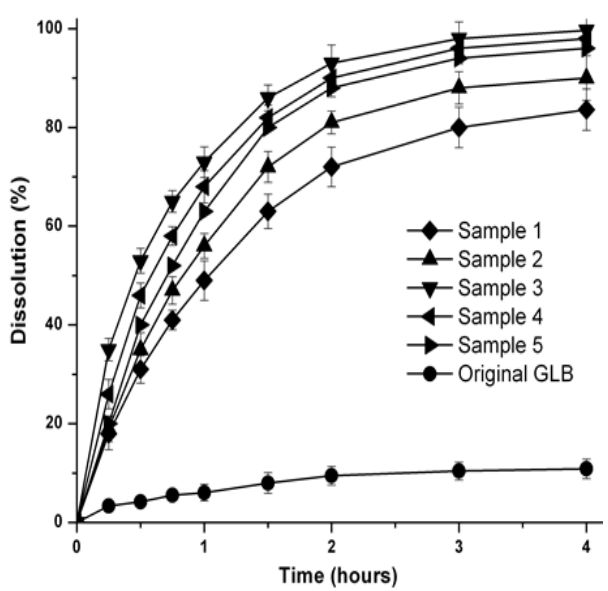

Figure 10: Dissolution profiles of original GLB and samples prepared by EPN.

substantial increase in the percent dissolution. As seen from Table 1, the $R^{2}$ value of more than 0.9 indicated good correlation between the Noyes-Whitney equation and our measured dissolution data, and the dissolution rate constant $K$ increased with the decrease in the particle size. The particle size of original GLB powder was reduced from 111.2 $\mu \mathrm{m}$ to a size in a range of approximately $120-226 \mathrm{~nm}$ by the EPN process along with the reduction in the crystallinity proved by the lower $\Delta \mathrm{H}_{\mathrm{f}}$ values shown in Table 1 . Both these factors resulted in an increased percent dissolution for the GLB nanopaticles by 7.7 to 9 times compared to the original GLB powder.

Overall, the EPN method developed is very simple but has the limitation of using organic solvents. Although the EPN method requires use of organic solvents but it should be noted that the amount of the residual solvents (ethanol and hexane) in the samples prepared by EPN was below the acceptable level for residual solvents in pharmaceuticals as determined by FDA for the safety of the patients. If the solvents used during the process can be recycled and reused economically, it will greatly increase the benefit of the method. Therefore, EPN represents an effective method to obtain uniform drug nanoparticles with a significantly higher rate of dissolution in-vitro. The scaling up would require a low-pressure set up for quick evaporation of the solvents. Large surface area of the set up and smaller batches of the nanosuspension would ensure faster evaporation and recovery of the nanoparticles. In comparison to the existing techniques for production of drug nanoparticles, the EPN method has the advantage of preparing drug nanoparticles without using any toxic surfactants or stabilisers.

The drug nanoparticles prepared can be used in the capsules or tablet form for oral drug delivery. The next step in our study is to evaluate the stability of these nanoparticles in the formulation. The particle size and zeta potential will be monitored over time for further studies.

\section{Conclusions}

This study demonstrated that the evaporative precipitation of nanosuspension (EPN) method is able to prepare nanoparticles of several poorly water soluble drugs such as silymarin, hesperetin and glibenclamide. The method developed is simple and cost effective. The process parameters, such as drug concentration and solvent to antisolvent volume ratio were investigated and optimized to produce the smallest drug nanoparticles. The drug nanoparticles prepared by the EPN method exhibited lower crystallinity compared to the original drug powder as revealed by the DSC analysis. The percent dissolution of drug particles depended on particle size and crystallinity. The EPN prepared particles of the drugs presented the considerable reduction in particle size and consequently the significant enhancement in the dissolution rate compared to the original drug powders. To conclude, EPN represents an efficient method to obtain uniform drug nanoparticles with a significantly higher rate of dissolution than original drug powders. The higher dissolution in-vitro can translate into an increased bioavailability in-vivo. The drug nanoparticles produced by this systematic method would have high potential for delivery in much smaller doses compared with commercial preparation containing the normal form of the drug.

\section{References}

1. Aghazadeh S, Amini R, Yazdanparast R, Ghaffari SH (2011) Anti-apoptotic and anti-inflammatory effects of Silybum marianum in treatment of experimental steatohepatitis. Exp Toxicol Pathol 63: 569-574.

2. Aranganathan S, Panneer Selvam J, Nalini N (2009) Hesperetin exerts dose dependent chemopreventive effect against 1,2-dimethyl hydrazine induced rat colon carcinogenesis. Invest New Drugs 27: 203-213.

3. Barzaghi N, Crema F, Gatti G, Pifferi G, Perucca E (1990) Pharmacokinetic studies on IdB 1016, a silybin- phosphatidylcholine complex, in healthy human subjects. Eur J Drug Metab Pharmacokinet 15: 333-338.

4. Choi EJ (2008) Antioxidative effects of hesperetin against 7,12-dimethylbenz(a) anthracene-induced oxidative stress in mice. Life Sci 82: 1059-1064.

5. Davis SN, Granner DK (2001) Insulin, oral hypoglycemic agents and the pharmacology of the endocrine pancreas. Goodman and Gilman's The pharmacological basis of therapeutics(10th ed) New York: McGraw Hill Professional, pp: 1679-1714.

6. des Rieux A, Ragnarsson EG, Gullberg E, Préat V, Schneider YJ, et al. (2005) Transport of nanoparticles across an in vitro model of the human intestinal follicle associated epithelium. Eur J Pharm Sci 25: 455-465

7. Formica JV, Regelson W (1995) Review of the biology of Quercetin and related bioflavonoids. Food Chem Toxicol 33: 1061-1080.

8. Fraschini F, Demartini G, Esposti D (2002) Pharmacology of silymarin. Clin Drug Invest 22: 51-65.

9. Galati EM, Monforte MT, Kirjavainen S, Forestieri AM, Trovato A, et al. (1994) Biological effects of hesperidin, a citrus flavonoid. (Note I): antiinflammatory and analgesic activity. Farmaco 40: 709-712

10. Hancock BC, Zografi G (1997) Characteristics and significance of the amorphous state in pharmaceutical systems. J Pharm Sci 86: 1-12.

11. Hörter D, Dressman JB (2001) Influence of physicochemical properties on dissolution of drugs in the gastrointestinal tract. Adv Drug Deliv Rev 46: 75-87.

12. Kakran M, Sahoo NG, Li L (2011) Dissolution enhancement of quercetin through nanofabrication, complexation, and solid dispersion. Colloids Surf B Biointerfaces 88: 121-130.

13. Kakran M, Sahoo NG, Li L, Judeh Z (2012) Fabrication of quercetin nanoparticles by anti-solvent precipitation method for enhanced dissolution. Powder Technol 223: 59-64.

14. Kakran M, Sahoo NG, Tan I-L, Lin Li (2012) Preparation of nanoparticles of poorly water-soluble antioxidant curcumin by antisolvent precipitation methods. J Nanopart Res 14: 757.

15. Kakran M, Sahoo NG, Li L, Judeh Z (2013) Particle size reduction of poorly water soluble artemisinin via antisolvent precipitation with a syringe pump. Powder Technol 237: 468-476.

16. Kanaze FI, Kokkalou E, Niopas I, Georgarakis M, Stergiou A, et al. (2006) Dissolution enhancement of flavonoids by solid dispersion in PVP and PEG matrixes: A comparative study. J Appl Polym Sci 102: 460-471.

17. Keck CM, Müller RH (2006) Drug nanocrystals of poorly soluble drugs produced by high pressure homogenisation. Eur J Pharm Biopharm 62: 3-16.

18. Kim JY, Jung KJ, Choi JS, Chung HY (2004) Hesperetin: a potent antioxidant against peroxynitrite. Free Radic Res 38: 761-769. 
Citation: Kakran M, Sahoo GN, Li L (2015) Fabrication of Nanoparticles of Silymarin, Hesperetin and Glibenclamide by Evaporative Precipitation of Nanosuspension for Fast Dissolution. Pharm Anal Acta 6: 326. doi:10.4172/2153-2435.1000326

19. Koziara JM, Lockman PR, Allen DD, Mumper RJ (2003) In situ blood-brain barrier transport of nanoparticles. Pharm Res 20: 1772-1778.

20. Kvasnicka F, Bíba B, Sevcík R, Voldrich M, Krátká J (2003) Analysis of the active components of silymarin. J Chromatogr A 990: 239-245.

21. Lai SK, Wang YY, Hanes J (2009) Mucus-penetrating nanoparticles for drug and gene delivery to mucosal tissues. Adv Drug Deliv Rev 61: 158-171.

22. $\mathrm{Li} \mathrm{FQ,} \mathrm{Hu} \mathrm{JH} \mathrm{(2004)} \mathrm{Improvement} \mathrm{of} \mathrm{the} \mathrm{dissolution} \mathrm{rate} \mathrm{of} \mathrm{silymarin} \mathrm{by} \mathrm{means}$ of solid dispersions. Chem Pharm Bull (Tokyo) 52: 972-973.

23. Ligtenberg JJ, Venker CE, Sluiter WJ, Reitsma WD, Van Haeften TW (1997) Effect of glibenclamide on insulin release at moderate and high blood glucose levels in normal man. Eur J Clin Invest 27: 685-689.

24. Lipinski CA, Lombardo F, Dominy BW, Feeney PJ (2001) Experimental and computational approaches to estimate solubility and permeability in drug discovery and development settings. Adv Drug Deliv Rev 46: 3-26.

25. Max JJ, Chapados C (2008) Infrared spectroscopy of methanol-hexane liquid mixtures. I. Free OH present in minute quantities. J Chem Phys 128: 224512.

26. Noyes AA, Whitney WR (1897) The rate of solution of solid substances in their own solutions. J Am Chem Soc 19: 930-934

27. Pepping J (1999) Milk thistle: Silybum marianum. Am J Health Syst Pharm 56: 1195-1197.
28. Pollard SE, Whiteman M, Spencer JP (2006) Modulation of peroxynitriteinduced fibroblast injury by hesperetin: a role for intracellular scavenging and modulation of ERK signalling. Biochem Biophys Res Commun 347: 916-923.

29. Pradhan SC, Girish C (2006) Hepatoprotective herbal drug, silymarin from experimental pharmacology to clinical medicine. Indian J Med Res 124: 491504

30. Sahoo NG, Abbas A, Li CM (2008) Micro/Nanoparticle design and fabrication for pharmaceutical drug preparation and delivery applications. Curr Drug Ther 3: $78-97$.

31. Sherif IO, Al-Gayyar MM (2013) Antioxidant, anti-inflammatory and hepatoprotective effects of silymarin on hepatic dysfunction induced by sodium nitrite. Eur Cytokine Netw 24: 114-121.

32. Srirangam R, Majumdar S (2010) Passive asymmetric transport of hesperetin across isolated rabbit cornea. Int J Pharm 394: 60-67.

33. Svenson S (2004) Overview: Carrier-Based Drug Delivery, Carrier-Based Drug Delivery, ACS Symposium Series Vol. 879, Washington DC: American Chemical Society pp: 2-23.

34. Ting HJ, Khasawneh FT (2010) Glybenclamide: an antidiabetic with in vivo antithrombotic activity. Eur J Pharmacol 649: 249-254. 